\title{
Eficacia del diagnóstico diferencial de tuberculosis mediante reacción de polimerasa en cadena en lesiones granulomatosas de tejidos fijados en formalina e incluidos en parafina
}

\author{
Sonia Montenegro, Carolina Delgado, Susana Pineda, Cristian Reyes, Tiare de la Barra, \\ Claudia Cabezas, Loreto Spencer y Francisco Mucientes
}

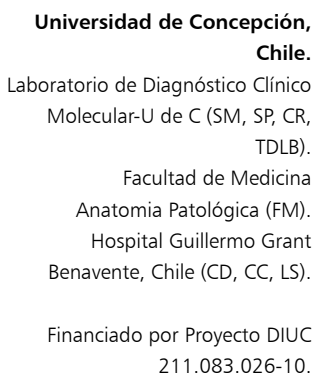

Universidad de Concepción Chile. Laboratorio de Diagnóstico Clínico Molecular-U de C (SM, SP, CR TDLB).

Facultad de Medicin Anatomia Patológica (FM). Hospital Guillermo Grant Benavente, Chile (CD, CC, LS)

Financiado por Proyecto DIUC 211.083.026-10.

Recibido: 23 de diciembre de 2013 Aceptado: 22 de octubre de 2014

Correspondencia a:

Sonia Montenegro Heredia labpatologiamolecular@gmail.com

\section{Efficacy of PCR for the differential diagnosis of tuberculosis in granulomatous lesions of paraffin-embedded formalin fixed tissues}

Background: Granulomatous lesions occur in tuberculosis (TB), other infections, toxic, allergic, and autoimmune diseases among others. In absence of a an acid-fast bacilli (AFB) confirmation of TB is necessary. Objective: To assess the efficacy of PCR for TB detection and to correlate with granuloma histology and AFB staining. Methods: We analyzed 380 fixed paraffin-embedded tissues (PETs) of granulomas with and without caseous necrosis; suppurative; sarcoidal; or of chronic nonspecific nature. Nested PCR-IS6110 for Mycobacterium tuberculosis complex (MTB) and a nested pan-Mycobacterium for the hsp65 gene were used for Mycobacterium spp detection. Results: PCR was more sensitive than AFB staining for all five catageories of granulomas: G1: PCR 71\%, AFB staining 28\%. G2: PCR 37\%, AFB 8\%. G3: PCR 17\%, AFB staining 7\%. G4: PCR 8\%, AFB staining 4\%. G5: PCR $6 \%$, AFB staining $0 \%$. Conclusions: Molecular diagnosis of TB using PCR-based testing is a fast, efficacious and sensitive method that increased the accuracy of PET histological diagnosis associated with granulomatous lesions.

Key words: PCR, baciloscopy, tuberculosis, granulomas, paraffin-embedded tissues.

Palabras clave: RPC, baciloscopia, tuberculosis, granulomas, tejido fijado e incluido en parafina.

\section{Introducción}

$\mathrm{E}$ 1 diagnóstico de tuberculosis (TBC) causada por el complejo Mycobacterium tuberculosis (MTB) debe ser rápido y confiable para iniciar un tratamiento oportuno, evitar complicaciones graves y prevenir la transmisión de la enfermedad ${ }^{1}$. Las lesiones granulomatosas características de TBC también ocurren en otras enfermedades infecciosas (brucelosis, lepra, enfermedad del rasguño de gato, en algunos hongos y virus) ${ }^{2}$ y no infecciosas (carcinomas, linfomas, sarcoidosis, cuadros tóxicos, alérgicos y autoinmunes) ${ }^{3-5}$. Estas entidades a su vez presentan una gran variedad de patrones histológicos con distintos tipos de granulomas como necrobióticos, tuberculoideos, supurativos, sarcoidales y a cuerpo extraño o crónico inespecífico ${ }^{6,7}$.

El granuloma infeccioso clásico o tubérculo es causado por MTB e histológicamente presenta un centro de necrosis caseosa rodeado de macrófagos epiteliodes activados, linfocitos, fibroblastos, células gigantes tipo Langhans y micobacterias. Este tipo de lesión permite un diagnóstico presuntivo confiable de $\mathrm{TBC}^{7,8}$. Sin embargo, en pacientes inmunocomprometidos y/o con VIH, la lesión tuberculosa aparece como granulomas poco definidos, no caseosos y con un variado espectro histopatológico ${ }^{7-9}$. Durante la infección tuberculosa, los macrófagos transportan los bacilos hacia los ganglios linfáticos regionales, desde donde pasan a la circulación y se depositan en áreas apicales del pulmón, en ganglios distantes y en otros órganos como riñones, intestino, meninges, huesos etc. ${ }^{10-12}$.

Debido a la variedad de la lesión granulomatosa, el diagnóstico histológico sólo puede ser sugerente pero nunca confirmatorio de TBC en ausencia de baciloscopia positiva (tinción Ziehl-Neelsen o auramina-O) ) $^{8,13}$. La baciloscopia es rápida y económica pero su baja sensibilidad (alrededor de $10^{4}$ bacilos para observación directa) ${ }^{8}$ determina que el cultivo sea esencial para un diagnóstico definitivo. Estos demoran semanas hasta identificar la especie, son de baja sensibilidad en muestras paucibacilares $\mathrm{y}$ es imposible hacerlo en tejidos fijados en formalina e incluidos en parafina (TFFP) ${ }^{8}$.

El diagnóstico molecular en base a RPC, en uso desde fines de la década de los 80 , mejoró significativamente el diagnóstico de $\mathrm{TBC}^{14-21}$ ya que su utilización directa en muestras clínicas combinó rapidez con especificidad y sensibilidad, con resultados considerablemente superiores a los obtenidos con baciloscopia ${ }^{15}$.

En TFFP, el diagnóstico molecular es una herramienta valiosa y útil para el diagnostico diferencial en presencia de lesiones granulomatosas con sospecha de TBC. El 
principal obstáculo en especímenes TFFP es que la fijación con formalina degrada el ADN, lo fragmenta, polimeriza $\mathrm{y}$ altera la secuencia ${ }^{22,23}$. Aunque este problema se ha reducido con el uso de formalina neutra o tamponada que al evitar la formación de ácido fórmico que desestabiliza y depurina el ADN, revierte la polimerización, si la fijación es superior a $24 \mathrm{~h}$ se puede llegar a un proceso irreversible de degradación del $\mathrm{ADN}^{23,24}$

La selección del blanco para la detección de MTB mediante RPC es importante y la secuencia de inserción IS6110 que presenta hasta 20 copias en TBC es una de las más utilizadas ${ }^{16,18-21}$. También se usan como gen blanco secuencias del gen 16S ARNr y del gen que codifica la proteína $65 \mathrm{kda}^{17}$. En trabajos previos hemos reportado la eficacia del diagnóstico molecular mediante RPC anidada IS6110 en muestras frescas ${ }^{18-24}$.

El propósito de este estudio fue determinar la eficacia de la RPC anidada IS6110- versus la baciloscopia (tinción de Ziehl-Neelsen/auramina O) para el diagnóstico diferencial de TBC en distintos tipos de lesiones granulomatosas a partir de TFFP.

\section{Pacientes y Métodos}

Analizamos 380 biopsias de pacientes, 52\% hombres (n: 196) con promedio de edad de 40 años y $48 \%$ mujeres (n: 184) con promedio de edad de 45 años. Los pacientes eran de tres grupos etarios: 39 niños (1-15 años), 269 adultos (16-60 años) y 66 adultos mayores ( $\geq 61$ años).

\section{Tipo de estudio y universo de la muestra}

Estudio retrospectivo/prospectivo de corte transversal. Un total de 285 biopsias retrospectivas (2004-2011) y 95 prospectivas recibidas durante la duración del estudio (2012 y 2013). Las biopsias eran de una variedad de tejidos de pacientes procedentes del Hospital Guillermo Grant Benavente (HGGB) de Concepción, que requerían de un diagnóstico diferencial de TBC.

\section{Clasificación histopatológica del granuloma}

El diagnóstico histológico fue realizado por un patólogo dedicado a la clasificación de los granulomas en cinco grupos: G1-granuloma tuberculoideo con necrosis caseosa; G2-granuloma tuberculoideo sin necrosis caseosa; G3-granuloma supurativo; G4-granuloma sarcoidal; G5-granuloma de tipo cuerpo extraño o inflamación crónica inespecífica. En el análisis rutinario los patólogos identifican el área de lesión granulomatosa para la microdisección manual del tejido para análisis de RPC.

\section{Baciloscopia en TFFP(Tinción Ziehl-Neelsen y auramina O)}

Realizada rutinariamente en el Laboratorio de Histología siguiendo protocolos estándar. Las tinciones fueron verificadas por un patólogo.

\section{Extracción de ADN a partir de TFFP}

Se procesaron 3-5 cortes seriados de $5 \mu \mathrm{m}$ y se realizó la microdiseción del área granulomatosa identificada. Las secciones fueron desparafinadas y rehidratadas de acuerdo a un protocolo estándar. El tejido fue digerido con proteinasa $\mathrm{K}$ y el $\mathrm{ADN}$ extraído utilizando un kit comercial Gentra/Qiagen ${ }^{\circledR}$ siguiendo las instrucciones del fabricante.

\section{Calidad del ADN de las muestras}

Fue verificada mediante RCP de un fragmento de $110 \mathrm{pb}$ con partidores del gen beta-globina ${ }^{25}$. Sólo se incluyeron en el estudio muestras con ADN amplificable.

\section{Controles positivos y negativos}

Como controles positivos se utilizó ADN extraído de cepas ATCC de MTB (H37rV), M. avium, M. bovis, $M$. intracellulare y de cultivos aislados localmente. Como controles negativos se utilizó ADN genómico humano comercial (Promega) y agua grado molecular (Life).

\section{Detección del complejo MTB y de otras micobacterias}

Se utilizaron RPC anidada -IS6110- de secuencias de la inserción IS6110 del genoma del complejo MTB que amplifican segmentos de 123 y de $92 \mathrm{pb}$ apropiados para muestras $\mathrm{TFFP}^{26,27}$. En muestras negativas para RPC anidada- IS6110 se realizó una pan-RPC anidada- $h s p 65^{26}$ que amplifica secuencias altamente conservadas del gen hsp65 presentes en la mayoría de las micobacterias. Las secuencias de los partidores utilizados se muestran en la Tabla 1.

RPC anidada-IS6110 para detección del complejo $M T B$. Consiste en dos RPC consecutivas (outer e inner), que amplifican un fragmento de $123 \mathrm{pb}$ o de $92 \mathrm{pb}$. La mezcla de reacción para RPC contiene: $1 \mathrm{X}$ buffer RPC; 1,4 $\mathrm{mM}$ de $\mathrm{MgCl}_{2} ; 16 \mathrm{mM}\left(\mathrm{NH}_{4}\right)_{2} \mathrm{SO}_{4} ; 0,2 \mathrm{mM}$ dNTPs; $0,625 \mathrm{U}$ de Taq polimerasa y $0,15 \mathrm{mM}$ de partidores outer y $0,3 \mathrm{mM}$ de partidores inner en un vol final de $10 \mu \mathrm{L}$. A la mezcla de RPC se agregan $3 \mu \mathrm{L}$ de ADN de la muestra (30-50 ng/ul) y de cada control positivo y negativo. El programa de termociclador consiste en: denaturación inicial a $94^{\circ} \mathrm{C}$ por $3 \mathrm{~min}$, con ciclos de $94^{\circ} \mathrm{C} / 30 \mathrm{~s}, 63^{\circ} \mathrm{C} / 35 \mathrm{~s}$ y $72^{\circ} \mathrm{C} / 35 \mathrm{~s}$ (20 ciclos en outer y 40 ciclos inner $)$, extensión final de $72^{\circ} \mathrm{C}$ por $7 \mathrm{~min}$. El producto de RPC inner se evalua mediante electroforesis en gel de agarosa y visualización en transluminador UV.

\section{Pan RPC anidada- hsp65 para Micobacteria spp.}

Como el anterior sólo que se realizan 30 ciclos outer y 35 ciclos inner y la temperatura de alineamiento y de extension es de $55^{\circ} \mathrm{C} / 1 \mathrm{~min}$ y de $72^{\circ} \mathrm{C} / 1 \mathrm{~min}$, respectivamente. 


\begin{tabular}{|c|c|c|c|c|}
\hline Gen & Partidor & Secuencias de partidores & Tamaño banda & Ref \\
\hline B-Globina & $\begin{array}{l}\mathrm{PCO} 3 \\
\mathrm{PCO} 4\end{array}$ & $\begin{array}{l}\text { 5' CAA CTT CAT CCA CGT TCA CC 3' } \\
\text { 5' ACA CAA CTG TGT TCA CTA GC 3' }\end{array}$ & $110 \mathrm{pb}$ & 25 \\
\hline IS6110 & $\begin{array}{l}\text { JS Out } \\
\text { KAS Out }\end{array}$ & $\begin{array}{l}\text { 5' CGG GAC CAC CCG CGG CAA AGC CCG CAG GAC 3' } \\
\text { 5' CAT CGT GGA AGC GAC CCG CCA GCC CAG GAT 3' }\end{array}$ & & 26 \\
\hline & $\begin{array}{l}\text { IS1 Inn } \\
\text { IS2 Inn }\end{array}$ & $\begin{array}{l}\text { 5' CCT GCG AGC GTA GGC GTC GG 3' } \\
\text { 5' CTC GTC CAG CGC CGC TTC GG 3' }\end{array}$ & $123 \mathrm{pb}$ & 26 \\
\hline & $\begin{array}{l}\text { IS3 Inn } \\
\text { IS4 Inn }\end{array}$ & $\begin{array}{l}5^{`} \text { TCC GGA CCA CCA GCA CCT AA 3`} \\
5^{`} \text { TCG GTG ACA AAG GCC ACG TA } 3 \text { ` }\end{array}$ & $92 \mathrm{pb}$ & 27 \\
\hline hsp65 & $\begin{array}{l}\text { MP1 out } \\
\text { MP2 out } \\
\text { MP3 inn } \\
\text { MP4 inn }\end{array}$ & $\begin{array}{l}\text { 5' AGG CGT TGG TTC GCG AGG G 3' } \\
\text { 5' TGA TGA CGC CCT CGT TGC C 3' } \\
\text { 5' CCA ACC CGC TCG GTC TCA A 3' } \\
\text { 5' CCG ATG GAC TGG TCA CCC 3' }\end{array}$ & $142 \mathrm{pb}$ & 26 \\
\hline
\end{tabular}

\section{Tabla 2. Detección de TBC mediante RCP y Baciloscopia según tipo de Granuloma en TFFP}

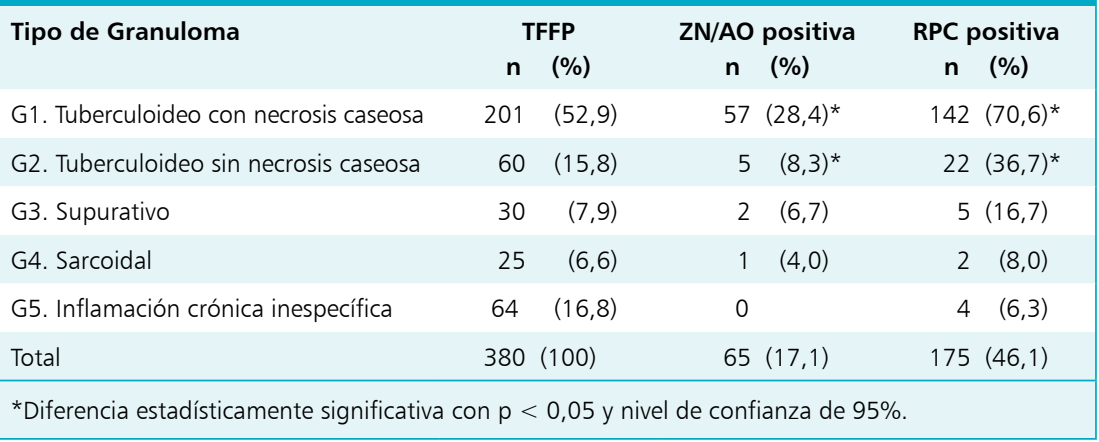

\section{Análisis estadístico}

Todos los datos obtenidos se analizaron estadísticamente con métodos univariables por comparación simple de medias muestrales por $\chi^{2}$ con un nivel de confianza del 95\% (StatGraphic).

\section{Resultados}

\section{Frecuencia de detección de TBC mediante RPC $y$ baciloscopia en muestras TFFP}

Del total de las muestras TFFP, $46,1 \%$ resultaron positivas para TBC mediante RPC y sólo $17,1 \%$ lo fueron en la baciloscopia (Tabla 2). El 95\% de las muestras positivas pertenecían al complejo MTB y sólo ocho de 175 muestras positivas lo fueron a otras especies de Mycobacterium.

En granulomas con necrosis caseosa (G1) que son caracteristicos de TBC, 70,6\% fueron positivas con RPC y $28,4 \%$ con baciloscopia, diferencia estadísticamente significativa $(p<0,05)$ (Tabla 2$)$. En granulomas sin necrosis caseosa $(\mathrm{G} 2)$ con lesiones sugerentes de TBC, se obtuvo $36,7 \%$ de positivos con RPC y $8,3 \%$ con baciloscopia, diferencias estadísticamente significativas $(\mathrm{p}<0,05)$ (Tabla 2). La positividad tanto de RPC y baciloscopia para detección de infección micobacteriana disminuyó fuertemente en lesiones granulomatosas que no eran histológicamente características de TBC como en granulomas de tipo supurativo, sarcoidal y crónico/ inespecífico y que además presentaban escasa evidencia clínica (Tabla 2).

\section{Frecuencia de biopsias positivas para TBC según género y edad}

En pacientes positivos para TBC por RPC, se observó que las variables género y edad fueron dependientes entre sí con un grado de confianza de 95\% $(\mathrm{p}<0,05)$. Hubo más positivos en hombres (53\%) que en mujeres (39\%) diferencia que fue estadísticamente significativa $(\mathrm{p}<0,05)$. Los diferentes grupos etarios presentaron frecuencias parecidas: $41 \%$ de positivos en niños; $48 \%$ en adultos y $39 \%$ en adultos mayores; sin embargo, hubo más hombres que mujeres positivos para TBC bajo 15 años de edad (52\% hombres y $21 \%$ mujeres) al igual que en edad adulta (57\% hombres y $39 \%$ mujeres) con diferencias estadísticamente significativas. En adultos mayores de 60 años, la mayoría de los positivos a TBC fue en mujeres ( $44 \%$ mujeres y $32 \%$ hombres), pero la diferencia no fue estadísticamente significativa.

\section{Desempeño de la RPC para la detección de TBC según antigüedad de la muestra}

Las muestras más antigüas tenían ocho años en archivo y su desempeño y calidad de ADN fue similar al de muestras más recientes. Se obtuvo $38 \%$ de positivas en TFFP 
Tabla 3. Frecuencia de biopsias positivas a tuberculosis según tipo de tejido y de lesión granulomatosa

\begin{tabular}{|c|c|c|c|c|c|c|c|c|c|}
\hline \multirow[t]{2}{*}{ Tipo tejido } & \multirow[t]{2}{*}{ n } & \multirow[t]{2}{*}{ (\%) } & \multirow{2}{*}{\multicolumn{2}{|c|}{$\begin{array}{l}\text { Baciloscopía positiva } \\
\qquad \quad(\%)\end{array}$}} & \multicolumn{2}{|c|}{ RPC positiva } & \multicolumn{3}{|c|}{ Granuloma predominante } \\
\hline & & & & & $\mathbf{n}$ & $(\%)$ & & $\mathbf{n}$ & (\%) \\
\hline Ganglio & 126 & $(33,2)$ & 20 & $(15,9)$ & 65 & $(51,6)$ & G1: & 83 & $(65,9)$ \\
\hline Piel & 49 & $(12,9)$ & 2 & $(4,1)$ & 12 & $(24,5)$ & G1: & 20 & $(40,8)$ \\
\hline Pleura & 43 & $(11,3)$ & 16 & $(37,2)$ & 31 & $(72,1)$ & G1: & 37 & $(86,0)$ \\
\hline Pulmón & 39 & $(10,3)$ & 7 & $(17,9)$ & 22 & $(56,4)$ & G1: & 24 & $(61,5)$ \\
\hline Intestino & 31 & $(8,2)$ & 8 & $(25,8)$ & 12 & $(38,7)$ & G2: & 14 & $(45,2)$ \\
\hline Tejido óseo & 17 & $(4,5)$ & 3 & $(17,6)$ & 7 & $(41,2)$ & G5: & 7 & $(41,2)$ \\
\hline Mama & 22 & $(5,8)$ & 0 & & 3 & $(13,6)$ & G2: & 13 & $(59,0)$ \\
\hline Otros Tejidos & 53 & $(13,9)$ & 9 & (17) & 23 & $(43,4)$ & G1: & 23 & $(43,4)$ \\
\hline Total & 380 & (100) & 65 & $(17,1)$ & 175 & $(46,1)$ & G1: 2 & 201 & $(52,8)$ \\
\hline
\end{tabular}

de ocho años de antigüedad, $52 \%$ con 7 años, $48 \%$ con dos años y $41 \%$ con de menos de un año. Estas diferencias no fueron estadísticamente significativas.

\section{Frecuencia de biopsias positivas para TBC según tipo de tejido y lesión granulomatosa}

Los tejidos más frecuentes analizados fueron ganglios linfáticos $(33 \%)$, piel $(13 \%)$, pleura $(11 \%)$ y pulmón $(10 \%)$. En el grupo de otros tejidos (n: 53$)$ se analizaron: testículo/próstata (n: 11), hígado/vesícula biliar (n: 9), cerebro (n: 7), riñón (n: 5), oral (n: 4), laringe (n: 4), útero (n: 4), tejido sinovial (n: 3), tiroides (n: 2), tejido cervical (n: 2) y tejido pericárdico (n: 2). En los ganglios linfáticos y en la piel que constituían $47 \%$ del total de muestras, predominó el granuloma tipo 1 con necrosis caseosa característico de TBC (Tabla 3).

Los tejidos extra-pulmonares más frecuentes fueron piel e intestino con granulomas tipo 1 y 2 , respectivamente. En mastitis granulomatosas, se determino la etiología de MTB en tres de 22 casos. Se pudo confirmar el diagnostico clínico BCGitis mediante RPC en siete casos y sólo dos por baciloscopia.

\section{Discusión}

En Chile la morbi-mortalidad por TBC ha disminuido debido a la efectividad de los programas de control, nuevos esquemas terapéuticos y a la vacunación masiva con $\mathrm{BCG}^{28}$. Sin embargo, aún continúa como un problema importante de salud pública sin lograrse una disminución de su incidencia, la que a nivel país es de 12,6 por 100.000 hab $^{29}$. En las dos primeras regiones de nuestro país, la tasa es mucho mayor ( 32 por 100.000 hab $)^{29}$ lo que se atribuye a la imigración desde Perú que, según informes de la OMS, tiene una prevalencia de TBC de 121 por $100.000 \mathrm{hab}^{30}$.
Otro agravante es el resurgimiento de la TBC por la co-infección con VIH, que complica y agrava el cuadro de TBC con presentación extrapulmonar, granulomas atípicos y dificultad de diagnosticar por métodos convencionales ${ }^{8,9,31}$ lo que tambien sucede en pacientes inmunosuprimidos por otras causas.

La ocurrencia de lesiones granulomatosas es muy común en patología, los agentes infecciosos son la etiología más frecuente y la más importante, la causada por micobacterias ${ }^{32,33}$. Los tejidos que presentan inflamación granulomatosa requieren de un rápido test confirmatorio a fin de obtener un diagnóstico concluyente, especialmente en pacientes con infección por VIH y/o inmunocomprometidos que padecen una enfermedad aguda y progresiva, con frecuencia fatal ${ }^{8,9}$.

Se han publicado diversos estudios en los que se reporta el uso de RPC en muestras de TFFP ${ }^{26,34-37}$; sin embargo, este es el primer estudio en el que se analiza una gran cantidad de muestras y en el que se incorpora una clasificacion del tipo de granuloma.

En los resultados que reportamos se puso en evidencia que $71 \%$ de las biopsias de tejidos con alta probabilidad de ser de origen tuberculoso presentaron RPC positiva en comparación con sólo $28 \%$ de las baciloscopias positivas. Nuestros resultados con muestras de TFFP fueron adecuados, se obtuvieron ADNs aceptables, lo que se puede atribuir a la selección de un blanco sensible, al uso de un método de RPC tipo anidada robusto, de alta sensibilidad y amplificación de fragmentos de pequeño tamaño (95-123 bp) que tienen mejor rendimiento en muestras TFFP parcialmente degradadas por efecto de la formalina ${ }^{22,27}$. La robustez del ensayo de RPC se vió reflejado en que no hubo diferencias significativas en el desempeño del test entre muestras recientes y de hasta ocho años de antigüedad.

Un tipo de TBC muy dificil de diagnosticar es la TBC pleural, debido a que el líquido pleural presenta escasa 
cantidad de bacterias y la baciloscopia puede llegar a tener un rendimiento muy bajo, con sólo $5 \%$. En este estudio, de 43 muestras de pleura con alta sospecha clínica de TBC, $72 \%$ resultaron positivas por RPC y $37 \%$ por la baciloscopia lo que demuestra la eficacia en el diagnóstico molecular.

La linfadenitis es la más común de las tuberculosis extrapulmonares ${ }^{9}$ y en este estudio, más de un tercio del total de muestras analizadas eran ganglios: cervicales, inguinales, axilares, mesentéricos y mediastinos. De las 126 muestras de ganglio analizadas, 83\% presentaron granuloma con necrosis caseosa central $(\mathrm{G} 1)$, característico de la lesión tuberculosa; de este grupo, 51,6\% fueron positivas por RPC y sólo $15,9 \%$ por baciloscopia. Estos resultados reflejan la sensibilidad y especificidad del diagnóstico molecular y su ventaja comparativa en relación al rendimiento de la baciloscopia.

La utilización de una técnica robusta de RPC permitió obtener una elevada correlación con las lesiones histológicas altamentes sugerentes de TBC y con resultados considerablemente superiores a los obtenidos mediante baciloscopia. La utilización de la RPC es de particular eficacia en infecciones micobacterianas paucibacilares y/o con presentación clínica e histológica poco característica, en los cuales el rendimiento de la baciloscopia es muy bajo.

Concluimos que:

- El diagnóstico molecular mediante el uso de un método de RPC robusto contribuye en forma importante con el diagnóstico histológico asociado a una respuesta tisular granulomatosa.

- Los resultados rápidos y confiables del diagnóstico de
MTB mediante RPC se traducirán en el inicio oportuno del tratamiento anti-tuberculoso.

- La utilización del método de RPC es un complemento de gran utilidad para el diagnóstico diferencial de MTB en biopsias fijadas en formalina e incluidas en parafina con diferentes tipos de lesiones granulomatosas.

\section{Resumen}

Introducción: Lesiones granulomatosas ocurren en tuberculosis (TBC), otras infecciones, condiciones tóxicas, alérgicas y autoinmunes, entre otras. Con baciloscopia negativa, es necesario confirmar el diagnóstico de TBC. Objetivo: Evaluar la eficacia de la RPC para detectar TBC comparado con baciloscopia en relación a la histología del granuloma. Métodos: Analisis de 380 tejidos fijados en formalina e incluidos en parafina (TFFP) con diferentes tipos de granulomas: con necrosis caseosa; sin necrosis caseosa; supurativo; sarcoidal; a cuerpo extraño/inespecífico. Utilizamos RPC anidada-IS6110 para detección del complejo Mycobacterium tuberculosis (MTB) y una pan-RPC anidada-hsp65 para Mycobacterium spp. Resultados: La detección de TBC mediante RPC fue significativamente superior a baciloscopia en los cinco tipos de granuloma: G1: RPC 71\%, baciloscopia 28\%; G2: RPC 37\%, baciloscopia 8\%; G3: RPC 17\%, baciloscopia 7\%; G4: RPC 8\%, baciloscopia 4\%; G5: RPC 6\%, baciloscopia $0 \%$. Conclusión: El diagnóstico de TBC por RPC es un método rápido, eficaz y de gran sensibilidad, que aumenta la precisión del diagnóstico diferencial de lesiones granulomatosas de TFFP procesados rutinariamente en histopatología.

\section{Referencias bibliográficas}

1.- Johansen I S, ThomsenV O, Forsgren A, Hansen B F, Lundgren B. Detection of Mycobacterium tuberculosis complex in formalin-fixed, paraffin-embedded tissue specimens with necrotizing granulomatous inflammation by strand displacement amplification. J Mol Diag 2004; 6: 231-5.

2.- James D G. A clinic-pathological classification of granulomatous disorders. Postgrad Med J 2000; 276: 457-65.

3.- Chakravorty S, Sen M K, Tyagi J S. Diagnosis of extrapulmonary tuberculosis by smear, culture and PCR using universal sample processing technology. J Clin Microbiol 2005; 43 (9): 4357-62.

4.- Collins T. Acute and chronic inflammation. In: Cotran RS, Kumar VK, Collins T, eds. Robbins Pathologic Basis of Disease. 6th ed. Philadelphia, Pa: Saunders Co; 1999; 50-88.
5.- Permi H S, Shetty J, Padma S K, Teerthanath S, Mathias M, Kumar S, et al. A histopathological study of granulomatous inflammation. Nitte Univ J Health Sc 2012; 2: 15-9.

6.- Weedon $\mathrm{D}$. The granulomatous reaction pattern. In: Weedon D (ed).Skin Pathology, 2nd ed.

7.- Zumia A, James G. Granulomatous infections: etiology and classification. Clin Infect Dis 1996; 23: $146-58$.

8.- Centers for Disease Control and Prevention. Trends in tuberculosis United States, 1998-2003. Morb Mortal Wkly Rep 2004; 53 (10): 209-15.

9.- Golden M, Vikram H. Extrapulmonary tuberculosis: an overview. Am Fam Phisician 2005; 72 (9): 1761-8.

10.- Gupta A, Kaul A, Tsolaki A, Kishore U, Bhakta S. Mycobacterium tuberculosis: immune evasion, latency and reactivation. Immunobiol 2012; 217: 363-74.

11.- Huynh K, Joshi S, Brown E. A delicate dance: host response to mycobacteria. Curr Opin Immunol 2011; 23: 1-9.

12.- Beqaj S H, Flesher R, Walker G, Smith S. Use of the real-time PCR assay in conjunction with MagNA pure for the detection of mycobacterial DNA from fixed specimens. Diagn Mol Pathol 2007; 16: 169-73.

13.- Chakrabarti A K, Halder K K, Das S, Chakrabarti S. Morphological classification of tuberculous lesions: preliminary observations. Ind J Tub 1994; 41: 139-42.

14.- Hsiao P F, Tzen C Y, Chen H C, Su H Y. PCR-based detection of Mycobacterium tuberculosis in tissues showing granulomatous inflammation without demonstrable acid-fast bacilli. Int J Dermatol 2003; 42: 281-6.

15.- Rattan A. PCR for diagnosis of tuberculosis:where are we now? Ind J Tub 2000; 47: 79-82.

16.- Eisenach K D, Cave M D, Bates J H, Crawford J T. PCR amplification of a 
repetitive DNA sequence specific for Mycobacterium tuberculosis. J Infect Dis 1990; 161: 977-81.

17.- Balasingham S V, Davidsen T, Szpinda I, Frye S A, Tønjum T. Molecular diagnostics in tuberculosis basis and implications for therapy. Mol Diagn Ther 2009; 13: 137-51.

18.- Dalovisio J R, Montenegro S, Kemmerly S A, Genre C F, Chambers R, Greer D, et al. Comparison of the amplified Mycobacterium tuberculosis (MTB) direct test, Amplicor MTB PCR, and IS6110-PCR for detection of MTB in respiratory specimens. Clin Infect Dis 1996; 23: 1099-106.

19.- Franchi L M, Cama R I, Gilman R H, Montenegro S, Sheen P. Detection of Mycobacterium tuberculosis in nasopharyngeal aspirate samples in children. Lancet 1998; 352: 1681-2.

20.- Caviedes L, Lee TS, Gilman R H, Sheen P, Spellman E, Montenegro S. Rapid, efficient detection and drug susceptibility testing of Mycobacterium tuberculosis in sputum by microscopic observation of broth cultures. J Clin Microbiol 2000; 38: 1203-08.

21.- Montenegro S, Gilman R, Sheen P, Cama R, Caviedes L, Hopper T, et al. Improved detection of Mycobacterium tuberculosis in Peruvian children by use of a heminested IS6110 polymerase chain reaction assay. Clin Infect Dis 2003; 36: 16-23.

22.- Zsikla V, Baumann M, Cathomas G. Effect of buffered formalin on amplification of DNA from paraffin wax embedded small biopsies using real-time PCR. Clin Pathol 2004; 57: 654-6.

23.- Schulz S, Cabras A D, Kremer M. Species identification of mycobacteria in paraffinembedded tissues: frequent detection of nontuberculous mycobacteria. Modern Pathol 2005; 18: 274-82.

24.- Hillemann D, Galle J, Vollmer E. Realtime PCR assay for improved detection of Mycobacterium tuberculosis complex in paraffin-embedded tissues. Int J Tuberc Lung Dis 2006; 10: 340-2.

25.- Saiki R, Gelfand D H, Stoffel S, Scharf S J, Higuchi R, Horn G T, et al. Primer-directed enzymatic amplification of DNA with a thermostable DNA polymerase. Science 1988; 239: 487-91.

26.- Marchetti G, Gori A, Catozzi L, Vago L, Nebuloni M, Rossi C, et al. Evaluation of PCR in detection of Mycobacterium tuberculosis from formalin-fixed, paraffin-embedded tissues: comparison of four amplification assays. J Clin Microbiol 1998; 36: 1512-7.

27.- Taylor G M, Goyal M, Legge A J, Shaw R J, Young D. Genotyping analysis of Mycobacterium tuberculosis from medieval human remains. Microbiol 1999; 145: 899-904.

28.- Subsecretaría de Salud Pública. Programa Nacional de Control de la Tuberculosis. Manual de Organización y Normas Técnicas. Minsal 2005.

29.- Minsal Chile 2013. Tasa de incidencia de tuberculosis en todas sus formas, por regiones, Chile 2000-2012. CIE 10: A15-9.

30.- World Health Organization (WHO). Global tuberculosis control 2013. WHO report 2013.

31.- Chaisson R E, Schecter G F, Theuer C P, Rutherford G W, Echenberg D F,
Hopewell P C. Tuberculosis in patients with the acquired immunodeficiency syndrome. Clinical features, response to therapy, and survival. Am Rev Respir Dis 1987; 136 (3): 570-4.

32.- Rey S. Enfermedades granulomatosas del pulmon. 2009. Parte I http://www.pathologyreview.com/sites/default/files/PDFs/conferences/ Enfermedades\%20Granulomatosas\%20del\%20 Pulmón.pdf

33.- Guler M, Simsek A, Ofluoglu R, Erguden H C, Çapan N. Are all granulomatous lesions tuberculosis? Respiratory Med 2012; 5: 42-4.

34.- Li J Y, Lo S T, Nag C S. Molecular detection of Mycobacterium tuberculosis in tissue showing granulomatous inflammation without demostrable acid-fast bacilli. Diag Mol Pathol 2000; 9: 67-74

35.- Park D, Kim J, Choi K, Lee J, Lee C, Sol M, et al. Comparison of PCR with histopathologic features for diagnosis of tuberculosis in formalin-fixed, paraffin-embedded histologic specimens. Arch Pathol Lab Med 2003, 127 326-30.

36.- Frevel T, Schäfer K L, Tötsch M, Böcker W, Dockhorn-Dworniczak B. PCR based detection of mycobacteria in paraffin wax embedded material routinely processed for morphological examination. Mol Pathol 1999; 52: 283-8.

37.- Vago L, Barberis M, GoriA. Nested PCR for Mycobacterium tuberculosis IS 6110 sequence on formalin-fixed paraffin-embedded tissues with granulomatous diseases for rapid diagnosis of tuberculosis. Am J Clin Pathol 1998; 109: 411-5. 